\title{
El coaching
}

\section{Autores:}

Jorge Cuadri Fernández

Sebastián Fierro Suero

Iván Palma Rodríguez

"La educación en valores y la práctica de actividad física en colectivos en situación de desigualdad". Grado en Ciencias de la Actividad Física y el Deporte. Universidad de Huelva.

Email: chan994@gmail.com 


\section{INDICE}

\section{Editorial}

1. En la clase anterior...

2. Conociendo al ponente...

3. Indagando sobre el coaching...

3.1 Artículo 1: Alejandro Arregui

3.2 Artículo 2: Javier Medrano

4. Aparición del coaching...
4.1 Teoría 1
4.2 Teoría 2

5. ¿Qué es el coaching?

6. Objetivos del coaching

7. El muñeco que apunta al éxito

8. Metodología del coaching:
8.1 El método GROW
8.2 La rueda de la vida

9. Competencias del coach

Licenciado en

Económicas,

Licenciado en

Ciencias Empre-

sariales y Marketing con Posgrado como Especialista en Economía

Bancaria

9.1 Parafrasear

9.2 La escucha activa

10. Actividades de clase

10.1 "50 Valores"

10.2 Dinámica escucha activa

11. El coaching más cerca de lo que creemos

12. Coaching deportivo: aplicación a la AF

13. Un pionero en el coaching deportivo: $W$. Timothy Gallwey

14. Conclusiones

15. Para saber más...

16. Bibliografía 
No hay nada más hermoso en esta vida que buscar tus límites
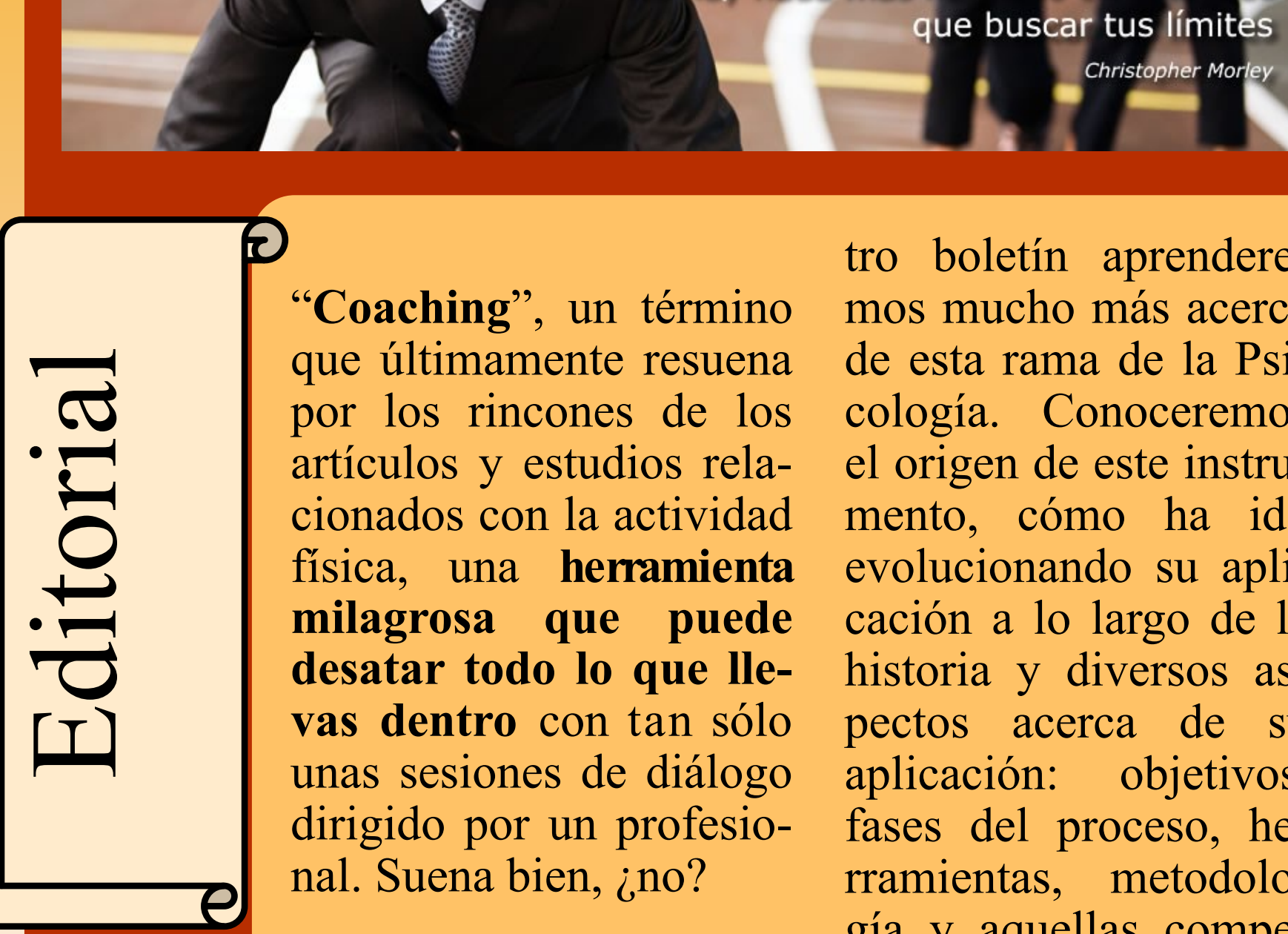

"Coaching", un término

tro boletín aprendereque últimamente resuena por los rincones de los artículos y estudios relacionados con la actividad física, una herramienta milagrosa que puede desatar todo lo que llevas dentro con tan sólo unas sesiones de diálogo dirigido por un profesional. Suena bien, ¿no?

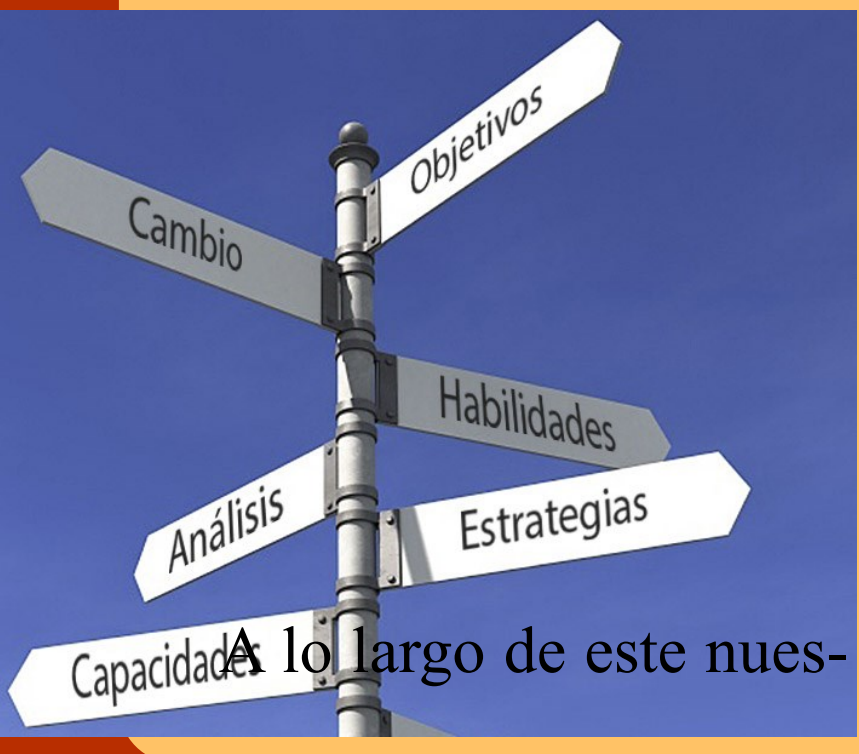
mos mucho más acerca de esta rama de la Psicología. Conoceremos el origen de este instrumento, cómo ha ido evolucionando su aplicación a lo largo de la historia y diversos aspectos acerca de su aplicación: objetivos, fases del proceso, herramientas, metodología y aquellas competencias que abarca, todo ello con el fin de romper las barreras que impiden al deportista sacar a relucir su máximo rendimiento. 
Coaching es desbloquear el potencial de la persona con el fin de maximizar su

rendimiento. Es ayudarles a aprender en vez de enseñar.

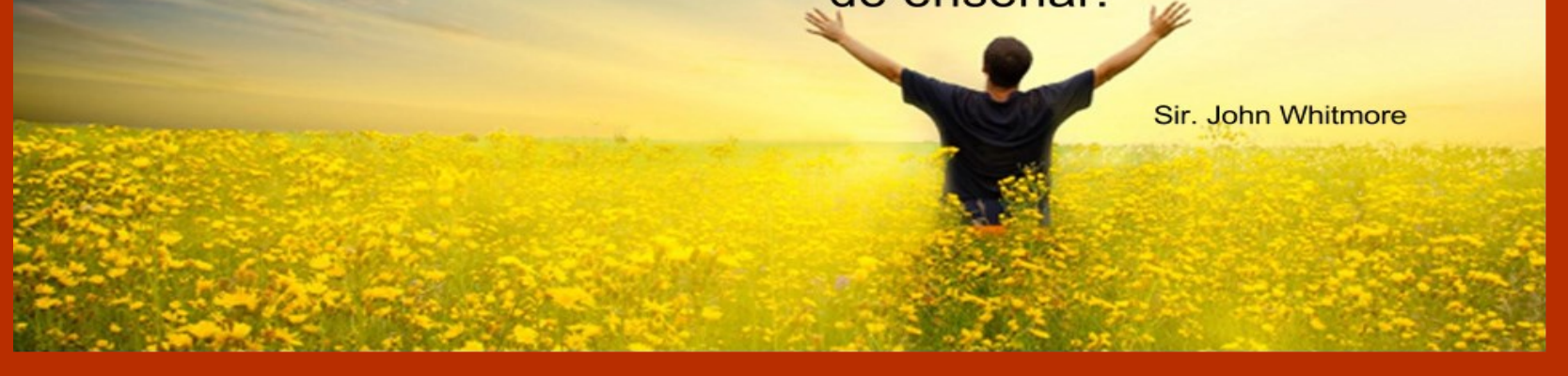

\section{El coaching, como vere-} mos posteriormente, ha regresado tras sus andanzas por el mundo empresarial a su ámbito de aplicación original, el mundo del deporte. Sin embargo, ¿estamos sacando el máximo partido a esta herramienta?¿Podríamos aplicarla fuera del alto rendimiento? ¿Tendría cabida en las clases de Educación Física? Estas incógnitas han ido ocupando nuestra mente a lo largo de la elaboración del boletín, y nos gustaría compartirlas con vosotros nuestros lectores desde el principio, para que Finalmente, antes de comenzar con la presentación de este práctico método de explotación del potencial oculto de la persona, os haremos un último adelanto: a lo largo de nuestra vida hemos tenido, tenemos y tendremos el coaching mucho más cerca de lo que jamás podríamos haber llegado a imaginar. Si no lo creéis, os lo mostraremos con ejemplos claros y que os resultarán curiosos y familiares, y os daremos la oportunidad de descubrirlos también por vosotros mismos.

intentéis buscarle respuesta como hicimos nosotros $\mathrm{y}$ plasmamos en uno de los apartados de este trabajo. 


\section{En la clase anterior...}

María Elena Bueno realizó al principio de la clase un resumen de la sesión anterior donde se pudo ver claro en todos los aspectos que se desarrollaron (contenido teórico, artículos y programas de intervención) que no es malo que afloren las emociones, sino que el problema llega cuando perdemos el control de estas. Estas emociones se manifiestan más intensamente durante la competición, en situaciones de oposición, y es importante mantener su control para que afecten lo menos posible al rendimiento. Además, las emociones influyen tanto positiva como negativamente en el estado anímico, y este, a su vez, influencia desde el ren- dimiento hasta el tiempo de recuperación de una lesión.

También se habló del entrenamiento mental como un medio para "engañar" a nuestro cerebro, ya que se ha demostrado que las respuestas neurofisiológicas de nuestro cerebro están condicionadas parcialmente por nuestros pensamientos (p.e. El efecto placebo, por el cual se hace creer al paciente que toma un medicamento que remediará su mal cuando en realidad este no tendrá ningún efecto sobre él y, sin embargo, se observa mejoría). En este ámbito también se analizó la técnica de visualización para la adecuada ejecución de un gesto técnico en el que está especializado el deportista, el triángulo dramático del que hay que intentar salir en situaciones de conflicto para alcanzar la madurez emocional, y que es importante realizar trabajo cognitivo para cuidar el cerebro de forma adecuada. desempeño.

Consiste en

ayudarle a

aprender en lugar

de enseñarle."

John Whilmore

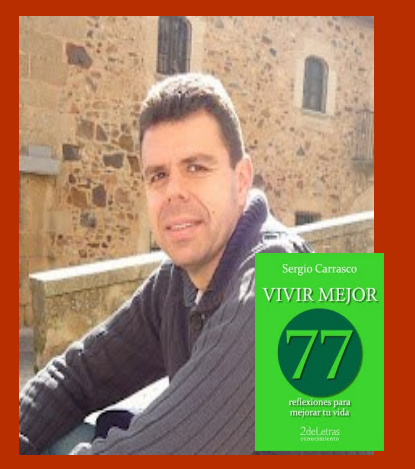

Sergio Carrasco Méndez

\section{Conociendo al ponente...}

Licenciado en Económicas, Licenciado en Ciencias Empresariales y Marketing con Posgrado como Especialista en Economía Bancaria. Posee una experiencia laboral de más de 14 años como directivo en diferentes empresas del sector financiero. En su em- presa actual desempeña las funciones de Responsable de Personas. Coach profesional (CPC n 10.347 por ASESCO). Máster en Coaching Personal y Máster en Coaching Ejecutivo. Socio fundador de Coworking Huelva (espacio de trabajo compartido para emprendedores).

Especialista en coaching y desarrollo personal. Entrenador Nacional de Baloncesto en categorías inferiores para el trabajo de la educación en valores desde la infancia. Risoterapeuta especialista en autoconocimiento y motivación. 


\section{Indagando sobre el coaching...}

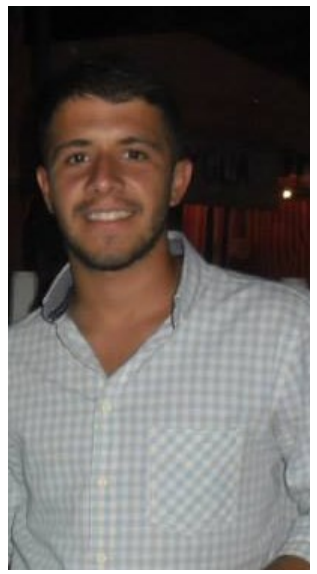

Alejandro Arregui Díaz

\section{I. Artículo I García-Naveira, A. (2013) “Aplicación profesional del coaching en el deporte: un estudio de caso único"}

El artículo expuesto por Alejandro da una visión general del coaching como un método que con el tiempo, después de pasar por el ámbito empresarial, ha vuelto a su lugar de origen, el ámbito deportivo. Numerosos autores, basándose en distintos aspectos de este método con fundamento en la Psi- cología, definen el coaching, del que podríamos decir que es un método en el cual, mediante preguntas, el coach consigue que el coachee descubra y explote por sí mismo su verdadero potencial. Además, en el artículo se exponen las características y la formación ideales para un coach, las cuales son inútiles sin la voluntad de cambio del cliente. Finalmente, se exponen las distintas fases del proceso de coaching, estructuradas en: generación del contexto y el vínculo, definición de la situación actual, definición de la situación ideal a alcanzar, generación de aprendizajes, desarrollo de un plan de acción, y paso

\subsection{Artículo 2 Gutiérrez, D. (20II) “Coaching deportivo para}

\section{entrenadores de fútbol"}

En el deporte de élite deportista confie en sí la preparación técnica mismo, mantenga la y física ha sido alcanza- mente gil para resolver da en cuanto a nivel de con eficiencia situaciones importancia por la for- dificiles y que requieren mación en otras disci- velocidad de reacción, y plinas, por la prepara- se encuentre motivado a ción psicológica, donde cambiar para conseguirentra en juego el lo. Para que se den camcoaching como un méto- bios conductuales en la do para lograr que el línea de trabajo del

\section{Aparición del coaching...}

\section{I. Teoría I}

El término tiene un origen anglosajón, ya que procede de la palabra "coach" entrenador, su desarrollo se relaciona con los primeros pensadores griegos $y$ entre ellos Sócrates (método socrático). En la actualidad, tomando como referente la importancia del entrenador como líder grupal, el "coaching" representa un sistema integral coach, es imprescindible que el coachee sea disciplinado y tenga voluntad de cambio. En este artículo, el proceso de coaching se estructura en cuatro fases: toma de conciencia de la realidad, querer cambiar, saber cómo cambiar y actuar.

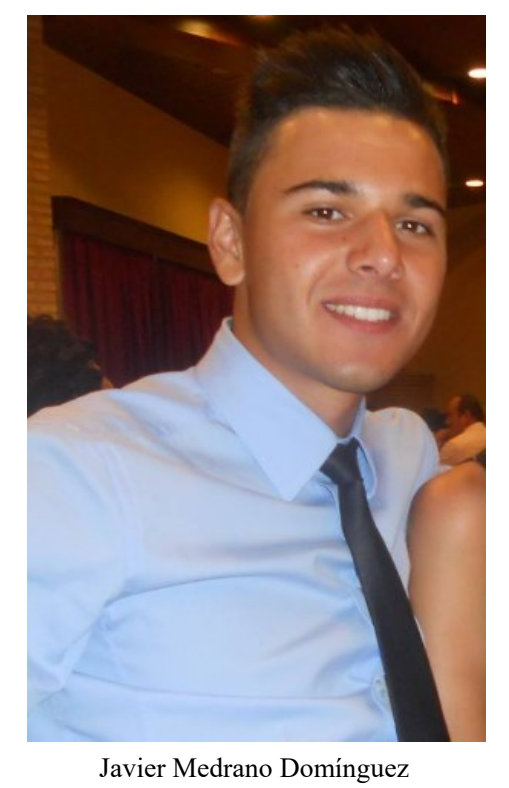

experimentado y aplicado con excelentes resultados en diferentes contextos, aportando los medios y recursos necesarios para la obtención del éxito (Fralile, 2013)

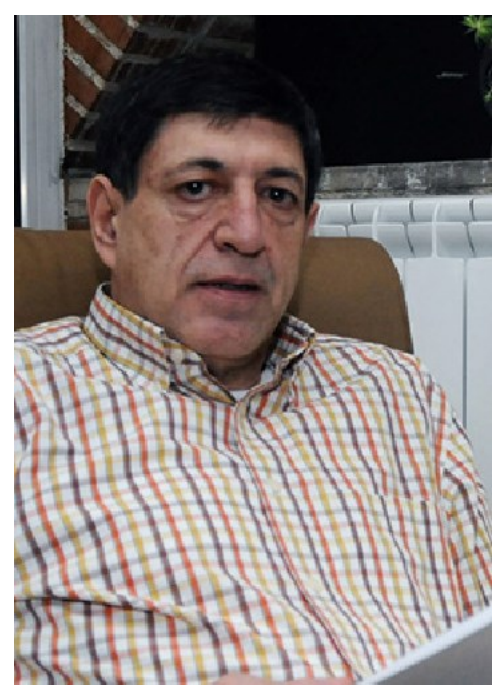

Antonio Fraile Aranda 


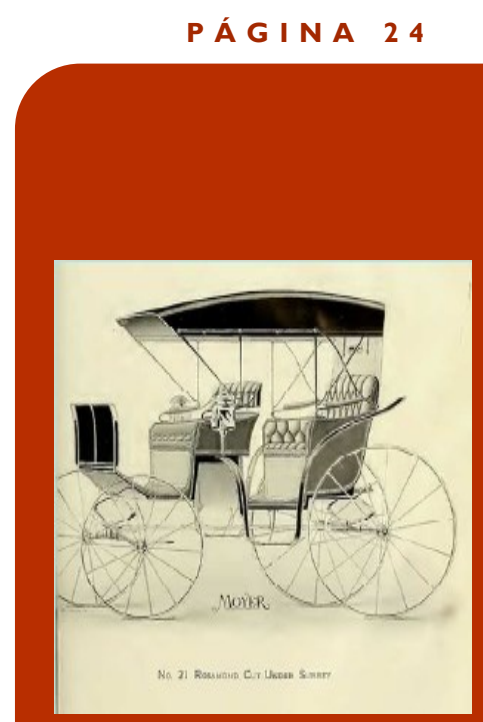

"El coaching es el arte de

crear un ambiente a través

de la conversación y de

una manera de ser, que

facilita el proceso por el

cual una persona se

moviliza de manera exitosa

para alcanzar sus metas

soñadas." "The Inner Game

of Tennis" Tim Galleway

Descubra la federación

internacional de Coah -

http://www.icf-es.com/mwsicf/ sobreicf/definicion-coaching-icf

-espana

\section{Aparición del coaching...}

\subsection{Teoría 2}

Tal como explicó nuestro ponente, existen otras corrientes defienden que el termino Coaching proviene de un pueblecito a unos $70 \mathrm{~km}$ de Hungría Ilamado Kocs.

Kocs sería un pueblo de transito donde se inventó una especie de carruaje que revolucionó el sistema de suspensión de la época. A este carruaje se le llamó "Kocsi" palabra que se traduce al alemán como Kutsche, al italiano como Cocchio, y al francés como Coach. Entonces, el término Coaching que en la actualidad utilizamos proviene de la palabra francesa Coach que significa "vehículo para transportar personas de un sitio a otro".

En idioma inglés también encontramos la palabra coach asociada a diferentes formas de transporte como pueden ser: Coach Driver (conductor); Coach Station (Estación de autobuses), etc.

Caminando un poco más por la historia nos encontramos a finales del siglo XVIII en Inglaterra, donde estos carruajes tirados por caballos se transformaron en un deporte practicado por la alta sociedad. Este nuevo deporte se Ilamó Coaching.

Por lo tanto, una rápida idea que podemos formarnos a partir de la información anterior nos ayudaría a entender el coaching como la manera de transportarnos hacia nuestras metas y objetivos con el mayor éxito posible y con la ayuda de nuestro mejor vehículo que sería la figura del Coach.

Igualmente podemos encontrar indicios de un proceso similar mucho antes, con Sócrates y su método filosófico interrogativo para buscar el conocimiento con la convicción que dentro de cada uno están las respuestas.

\section{5. ¿Qué es el Coaching?}

\section{- INTERNATIONAL COACH FEDERATION}

"El coaching profesional consiste en una relación profesional continuada que ayuda a obtener resultados extraordinarios en la vida, profesión, empresa o negocios de las personas. Mediante el proceso de coaching, el cliente profundiza en su conocimiento, aumenta su rendimiento y mejora su calidad de vida."

\section{- ESCUELA EUROPEA DE COACHING}

"Coaching es el arte de hacer preguntas para ayudar a otras personas a través del aprendizaje en la exploración y el descubrimiento de nuevas creencias que obtienen como resultados el logro de los objetivos"

\section{- ASESCO (ASOCIACIÓN ESPAÑOLA DE COACHING)}

"El coaching profesional es un proceso de entrenamiento personalizado y confidencial mediante un gran conjunto de herramientas que ayudan a cubrir el vació existente entre donde una persona está ahora y donde se desea estar. En la relación de Coaching el coach ayuda al desarrollo personal elevando la consciencia, generando responsabilidad y construyendo autoconfianza." 


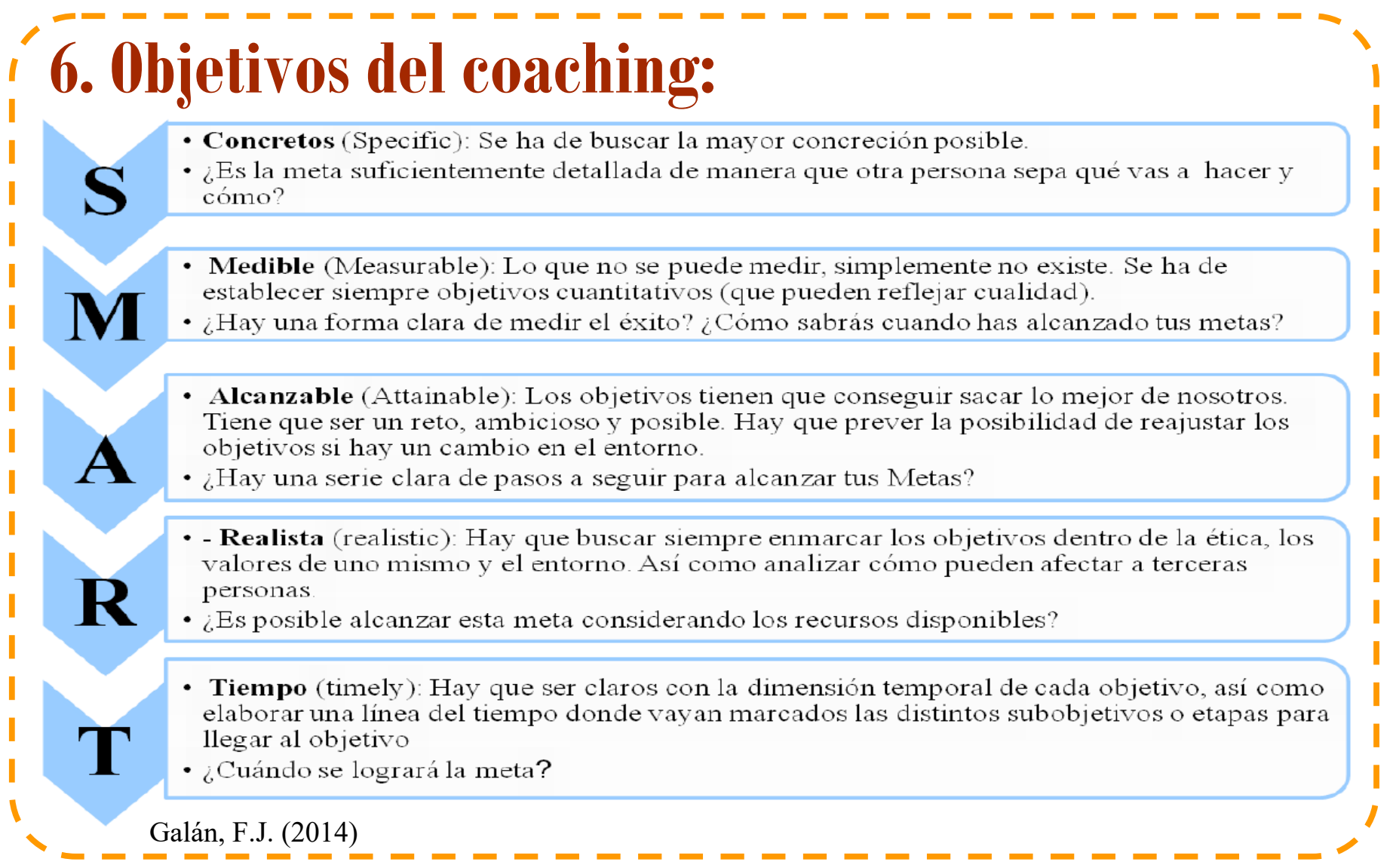

\section{El muñeco que apunta al éxito...}

Según Carrasco (2014) en una labor de coaching es fundamental: Saber, hacer y querer.

Con ellos construimos lo que Sergio nos presenta como un muñeco. El la labor de coaching hay que hacer que el cliente detecte aquello que le perjudica, que le limita, (basura) y lo eche fuera, lo elimine.

Por otro lado, tiene que marcar unos objetivos que deben ser asequibles, alcanzables, motivantes y suponer un reto para que sean motivantes.

Una vez tenemos lo que queremos conseguir, hay que pasar a la acción. Hay que encontrar el equilibrio entre la víctima y el súper héroe como nos presentaba Sebastián Fierro en el triángulo dramático en la clase anterior.

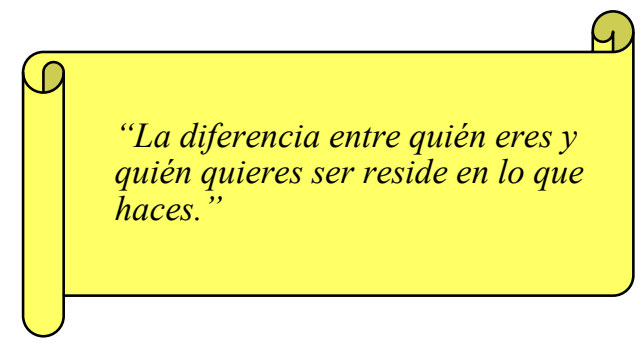

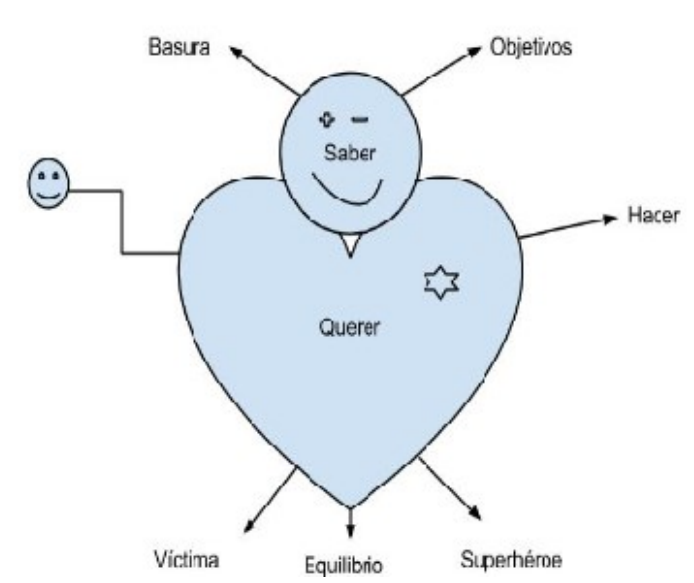

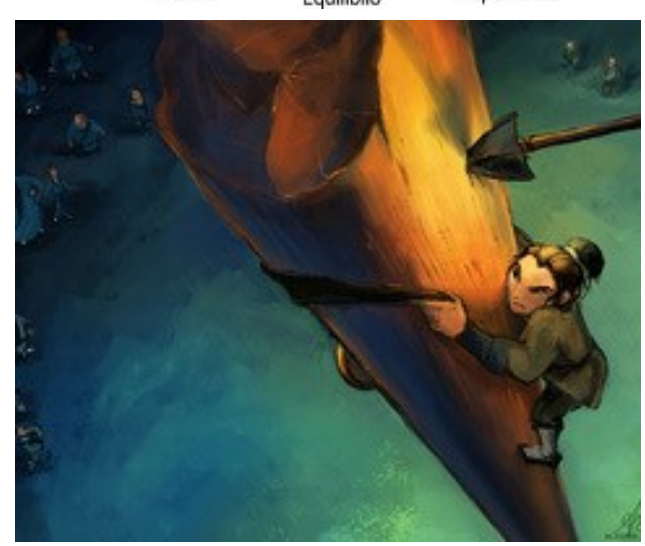




\section{La metodología del coaching}

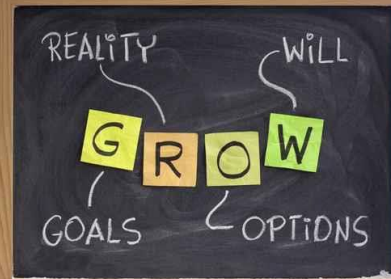

"Hay que

buscar el buen

equilibrio en el

movimiento $y$

no en la

quietud"

〜Bruce Lee
Visita el blog de David Prieto:

"Mi proposito de vida es guiar a las personas hacia la

libertad y la riqueza."

\subsection{El método GROW:}

El método de actuación en coaching es conocido por las siglas GROW, que a su vez sirve de regla nemotécnica y recordatorio de los objetivos de esta disciplina, "el crecimiento" de la persona. En su artículo, Galán (2014) lo explica de la siguiente manera:

$\mathbf{G}=$ Goal (objetivo), tiene que tener una serie de características: que sean medibles, concretos, alcanzables, retadores (que conlleven salir de la zona de confort), temporal (fijarlo en el tiempo) $o$ ecológico (solo los más puristas lo añaden. Hacen referencia a que esté acorde con los valores de tu vida, que no se salga de tus creencias, preferencias, etc.). Las preguntas del coach serán: ¿Cuál es tu reto?, ¿Cuál es tu objetivo?, ¿cómo sabrás que has alcanzado tu meta?

$\mathbf{R}=$ Reality Explorar tu realidad, ver en qué situación verdaderamente te encuentras. Esto se puede hacer mediante técnicas o herramientas como la rueda de la vida. Las preguntas del coach serán: ¿Cuál es tu situación actual?, ¿Cuánto te queda para alcanzar tu objetivo?, ¿Qué dificulta tu camino?
$\mathbf{O}=$ Opciones, ya sabemos lo que queremos conseguir, y ahora la pregunta es cómo y cuándo lo voy a conseguir. Aquí es donde se produce la búsqueda interior, se generan muchísimas emociones y de todas ellas tenemos que buscar la que verdaderamente le sirva a él para su forma de vida. Las preguntas del coach serán: ¿De qué opciones dispongo?, ¿cuáles son las alternativas?, ¿Cuáles son los pros y los contras de cada opción?, ¿Qué factores tienes que tomar en cuenta?

$\mathbf{W}=$ Will (la voluntad). Una vez que tie-nes tus objetivos, tienes punto de partida y tienes todas las opciones llega la pregunta: ¿es esa la verdadera meta que quieres? ¿Es esa la de verdad? de 0 a 10, ¿cómo es el compromiso con ella? si es menos de $10 \mathrm{n}$ te vale, tienes que saber qué es lo que tienes que hacer, el coach te puede enseñar cómo, pero no puede hacer que tu compromiso sea de 10. Las preguntas del coach serán: ¿Cuáles serán los siguientes pasos?, ¿Cuándo empezarás? ¿Cómo medirás tus avances? ¿Cómo harás para superar los obstáculos que se te puedan presentar? ¿Qué acción vas a adoptar? ¿Qué

\subsection{Rueda de la vida:}

Según Prieto (2013) la Rueda de la Vida te ayuda a valorar tu grado de felicidad en los distintos apartados de tu vida y de este modo conocer cuáles son las áreas que necesitas potenciar, desarrollar o mejorar. Se compone de ocho categorías que representan todos los aspectos a desarrollar como seres humanos, las áreas donde nos movemos y que determinan nuestro nivel de realización $\mathrm{y}$ felicidad.

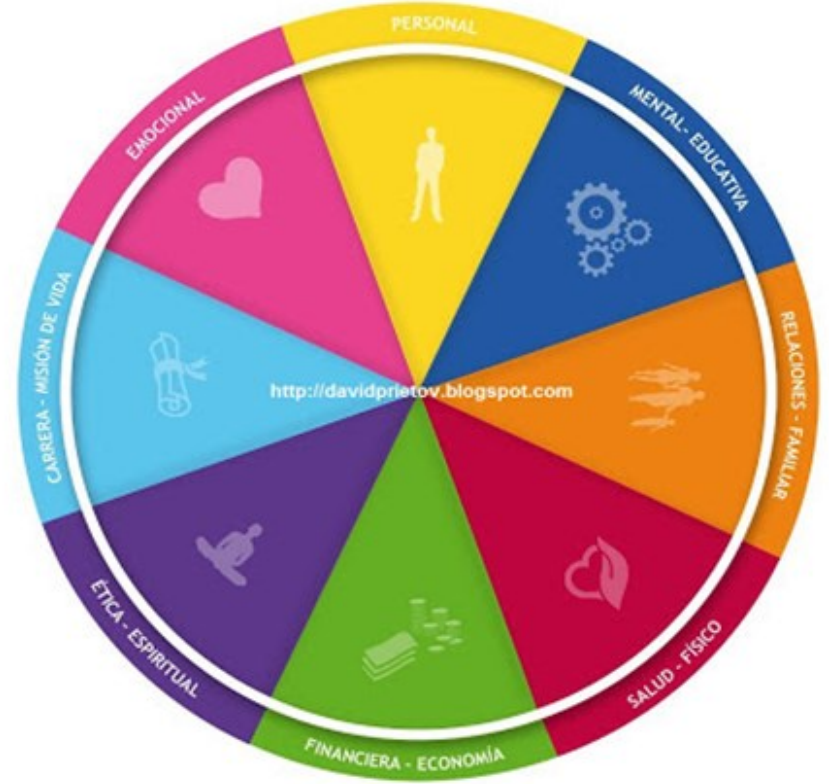

http://davidprietov.blogspot.com.es/2013/03/la-rueda-de-la -vida.html 


\section{Trata estos 8 apartados:}

1) Salud-Físico, que incluye todo lo relacionado con el cuerpo físico, la salud, la nutrición, el deporte, el bienestar y la belleza.

2) Personal, que incluye el aprendizaje, el crecimiento personal y el auto-desarrollo

3) Relaciones-Familiar, que incluye a los amigos, la familia y la sociedad. Aquí se evaluarán las relaciones que tienes en el ámbito privado y personal.

4) Mental-Educativa donde se engloba todo lo relacionado con el desarrollo de tus capacidades mentales.

El objetivo de esta área es despertar las facultades innatas de la mente. Partiendo de tus capacidades y desarrollo actual
5) Emocional donde se engloba el amor, la pareja, el equilibrio emocional y la sexualidad

6) Financiera-Economía donde entra todo lo relacionado con el dinero y las finanzas.

7) Ética-Espiritual cuya sección no se refiere necesariamente a la religión, aquí cabe destacar nuestro código de conducta, autoestima y el desarrollo espiritual.

8) Carrera-Misión de vida donde están incluidos todos los aspectos relacionados con el trabajo, la profesión, los estudios, la vocación, etc.

La clave de la felicidad es el equilibrio. Muchas veces, cuando nos sentimos insatisfechos a - pesar de tener éxito en alguna área, pensamos que necesitamos más éxito en esa misma área, | cuando lo que requerimos es equilibrar nuestra vida. La Rueda de la Vida nos servirá cómo indicativo de los puntos de tu vida en los que puedes mejorar.

\section{Competencias del coaching}

\subsection{Parafrasear}

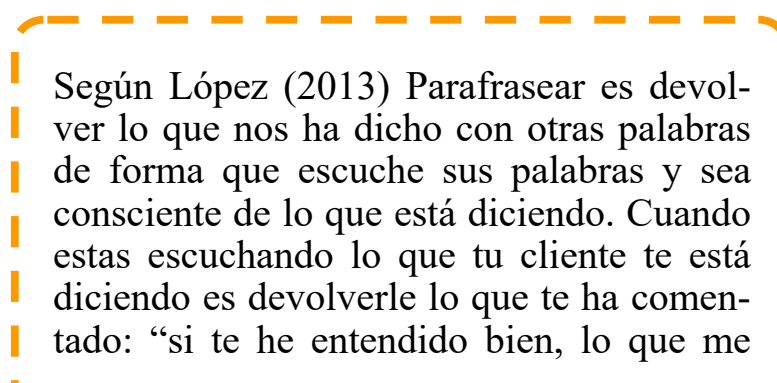

1

\subsection{La escucha activa}

Nuevamente, López (2013) nos comenta que "Es una atención plena en la persona que tienes delante, para tomar toda la información que tienes de ella. No estar esperando a que la persona termine para saber lo que decirle, sino tener la mente en blanco en ella y cuando acabe tener un feedback. No ir pensando qué le vas a responder mientras te habla."

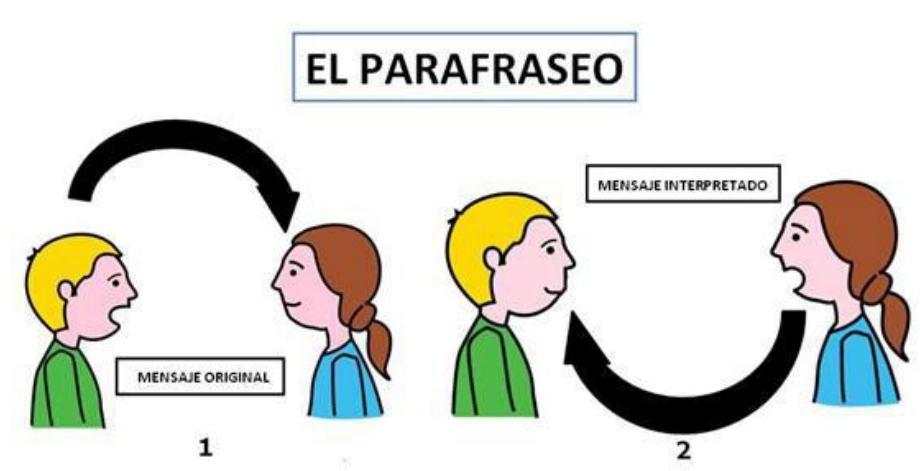

1.- Observar bienestar: Tono de voz, actitudes, gestos, poses, mímicas, expresión y mirada.

2.- Preguntarse: hacerse las siguientes preguntas para descubrir el verdadero significado de lo que el interlocutor quería decir.

Un gran principio de la escucha activa es ser muy consciente del otro. Para conseguirlo hay que:

\section{3.-Hacerle precisar los hechos:}

"Creo entender que lo que intentas decirme es que...", "Me parece que quieres decir que..." 


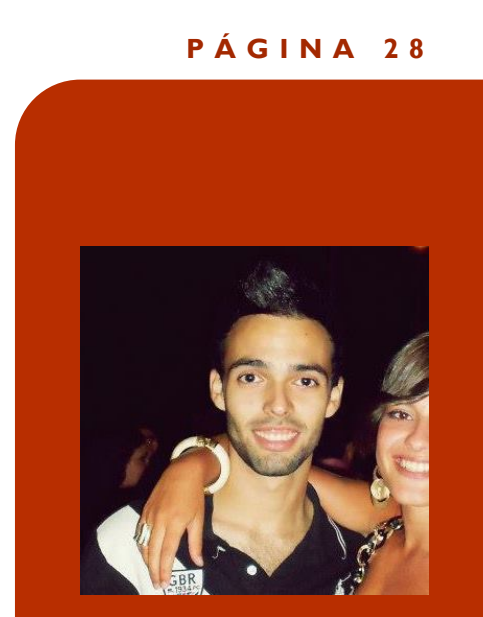

\section{Iván Palma}

"El coaching cubre el vacío existente entre lo que es ahora y lo que deseas ser. Es una relación profesional con otra persona que aceptará sólo lo mejor de ti y te aconsejará, guiará y estimulará para que

vayas mas allá de las

limitaciones que te impones a ti mismo y realices tu pleno potencial." "Coaching para el éxito" Talane Miedaner

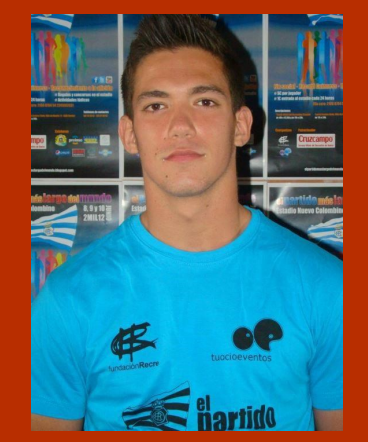

Jorge Cuadri

\section{Actividades de clase...}

- $\quad$ Elija 10..

- $\quad$ Elija 5..

- $\quad$ Elija 3...

"Este ejercicio nos muestra que si el plan de acción del coaching va en contra o chirría con alguno de los tres valores que hemos elegido en la lista de valores, hay un porcentaje altísimo de que acabemos dejando lo que estamos haciendo y que por lo tanto, el coachee deje a su cliente. Como ya hemos mencionado, si no existe voluntad de cambio o si no se sabe qué es lo que se quiere, la técnica del coaching no sirve para nada."
10.1. 50 valores...

\begin{tabular}{|l|l|l|l|}
\hline \multicolumn{5}{|c|}{ LISTA DE VALORES } \\
\hline Alegría & Creatividad & Fuerza & Poder \\
\hline Afecto & Crecimiento & Generosidad & Precisión \\
\hline Apego(amistad,fa & Cuidado & Gentileza & Productividad \\
\hline Apoyo & Cultivo & Gratitud & Progreso \\
\hline Aprendizaje & Cumplimiento & Honestidad & Realización \\
\hline Armonía & Desapego & Humildad & Respeto \\
\hline Autenticidad & Desarrollo & Humor & Romance \\
\hline Aventura & Disciplina & Igualdad & Sabiduría \\
\hline Belleza & Educación & Independencia & Salud \\
\hline Bienestar & Empatía & Integridad & Seguridad \\
\hline Bondad & Entrega & Justicia & Sencillez \\
\hline Claridad & Entusiasmo & Lealtad & Serenidad \\
\hline Colaboración & Equilibrio & Libertad & Servicio \\
\hline Compañerismo & Espiritualidad & Limpieza & Sinceridad \\
\hline Comprensión & Estabilidad & Madurez & Solidaridad \\
\hline Comunicación & Estética & Naturaleza & Ternura \\
\hline Comunidad & Exactitud & Nobleza & Tolerancia \\
\hline Conexión & Exito & Paciencia & Tradición \\
\hline Confianza & Familiar & Participación & Tranquilidad \\
\hline Constancia & Fidelidad & Pasión & Valentía \\
\hline Contribución & Flexibilidad & Paz & Variedad \\
\hline Cooperación & Franqueza & Paz interior & Vitalidad \\
\hline
\end{tabular}

"Instituto Europeo de Coaching"

\subsection{Dinámica escucha activa}

Para trabajar la importancia de la escucha activa, muy importante en el coaching, hicimos una dinámica en la que, por parejas, uno comenzaba a contar a otro una experiencia personal de gran importancia para él, mientras que el otro lo cortaba
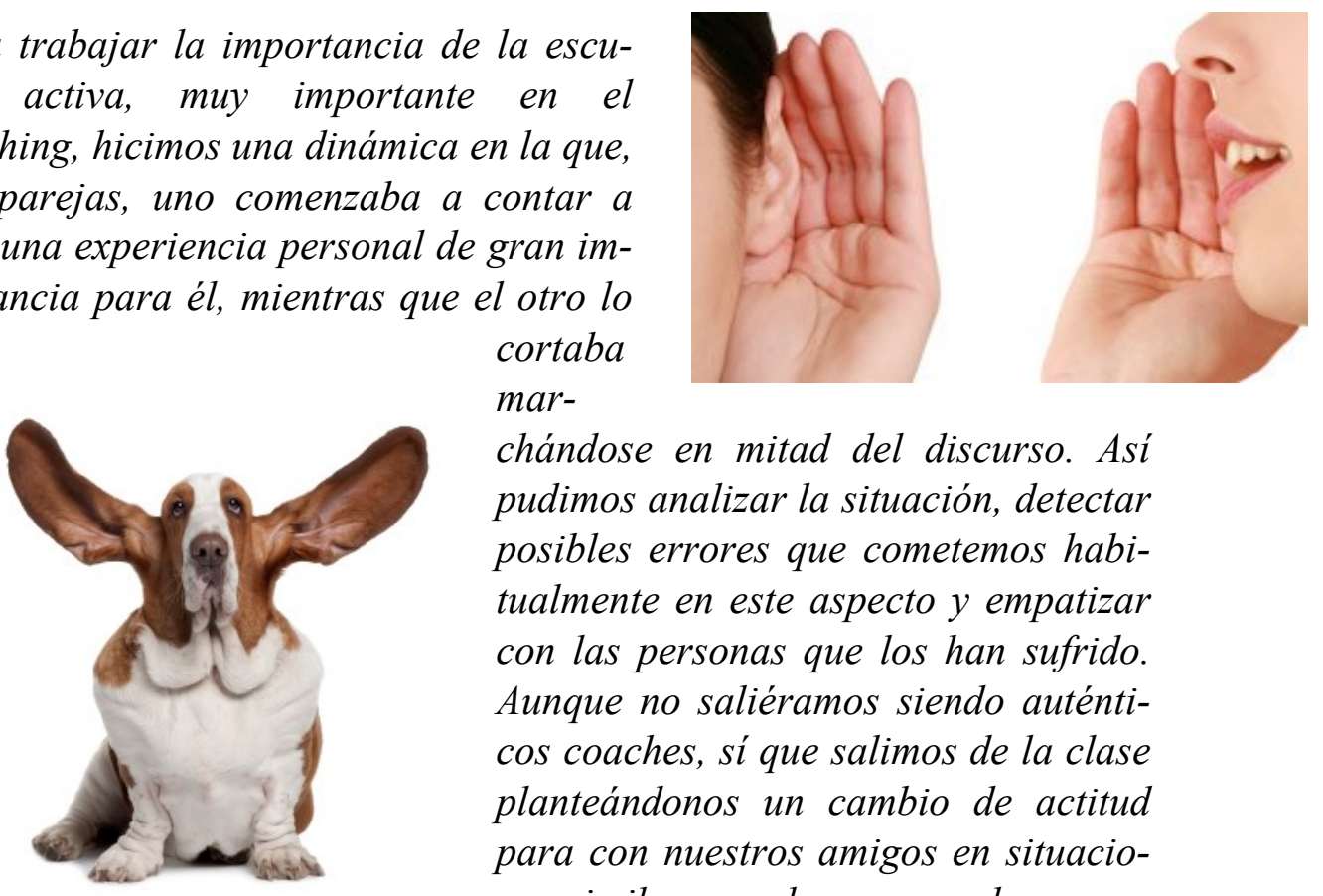
mar-

chándose en mitad del discurso. Así pudimos analizar la situación, detectar posibles errores que cometemos habitualmente en este aspecto y empatizar con las personas que los han sufrido. Aunque no saliéramos siendo auténticos coaches, sí que salimos de la clase planteándonos un cambio de actitud para con nuestros amigos en situaciones similares en las que muchas veces nuestro comportamiento no es el más acertado y no nos damos cuenta. 


\section{El Coching más cerca de lo que creemos:}

\section{Vídeo 1}

Para explicar qué es el Coaching, Sergio nos puso un pequeño video sobre la película La leyenda de Bagger Vance.

En ella el calif, actúa como un verdadero coach haciendo ver a su jugador más allá de "el campo" haciendo que mentalice sus objetivos, los encuentre; busque la forma de como alcanzar esos objetivos y la encuentre; que la armonía se funda con su alma para conseguir aquello que quiere.

El papel de calif es interpretado por el fantástico actor Will Smith quién actúa en todo momento como coach ya que consigue que su "cliente" Bagger Vance se reencuentre consigo mismo para sacar lo mejor de sí. Finalmente cuando Will Smith considera que ya no le necesita, abandona y se marcha, sin más. Ha conseguido su objetivo, llevar a su cliente hasta donde quería, hasta su propio yo.

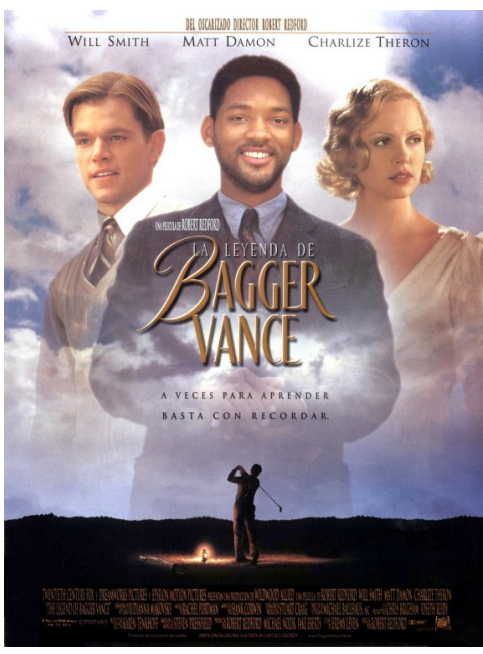

"...Yo no puedo llevarle allí, solo espero poder ayudarle a encontrar el camino. Está usted, esa bola, esa bandera y todo lo |
que es usted..."
Recuerde el víde0: https://www.youtube.com/watch?v=Kidd1KpyHYY
|

\section{Vídeo 2}

En el vídeo, Alicia se encontraba ante una encrucijada en la que podía tomar multitud de direcciones, ante lo cual decidió pedir ayuda al gato. Este le dijo que realmente no importaba qué camino escogiese, todos la llevarían a algún sitio; lo que tenía que pensar detenidamente era si camino que escogería la llevaría a donde realmente quería llegar.

\section{Vídeo 3 Véalo aquí:}

https://www.youtube.com/watch?

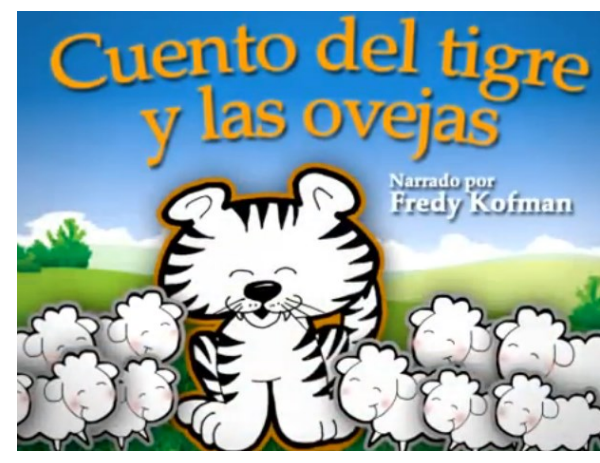

Llevado al campo del coaching, sacaríamos en conclusión que a la hora de pensar, actuar, decidir, tenemos que pensar si lo que hacemos hoy supone un paso más hacia la meta que queremos conseguir. Si no es así, estamos haciendo las cosas mal.

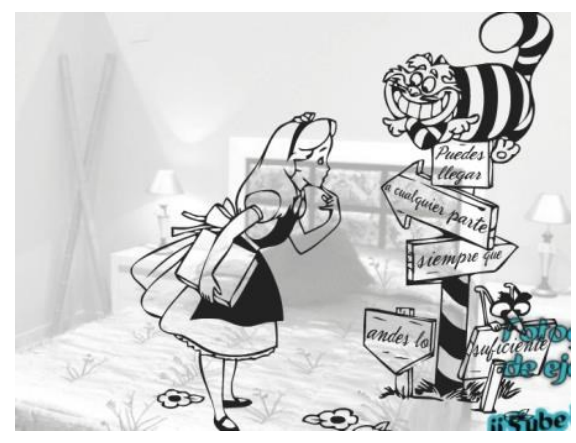

Un pequeño tigre que queda huérfano al morir su madre es acogido por un rebaño de ovejas, el cual lo cría como si fuera una más.

Un día, un tigre adulto que vio al pequeño tigre en medio del rebaño de ovejas comportándose como tal, lo llevó a un lago cercano para mostrarle su reflejo, medio del rebaño de ovejas comportándose como tal, lo llevó a un lago cercano para mostrarle su reflejo, para ense- ñarle que no era una oveja como él creía, sino que realmente era un tigre, un animal que corona la cadena alimentaria. Llevando esto al ámbito del coaching, podríamos extraer como conclusión que muchas veces nos creemos algo que no somos, no somos realmente conscientes de nuestro potencial, y eso nos limita y afecta a nuestra vida. 


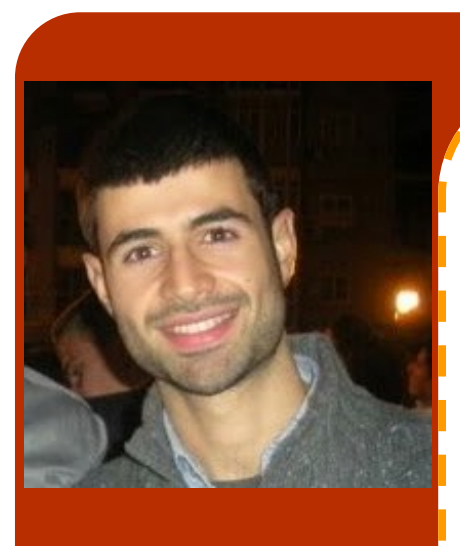

Sebastián Fierro Suero

"Incluso la gente que afirma que no podemos hacer nada para cambiar nuestro destino, mira antes de cruzar la calle". Stephen Hawking

\section{Coaching deportivo: aplicación a la Actividad Física}

El "coaching" aplicado al ámbito deportivo se puede definir como una técnica metodológica que facilita un proceso de formación y capacitación personal, entre educador y jugador, posibilitando que ambos puedan mejorar su autoconocimiento. Es decir, gracias a que nos conocemos mejor y nos relacionamos mejor con los demás, podremos actuar de forma más eficiente (tanto a partir de la actividad formativa del educador, como de aprendizaje del jugador). (Fralile, 2013).

Como se ha podido ver en los artículos expuestos y en la información proporcionada hasta ahora, el coaching se aplica en el ámbito del deporte de élite para ayudar a que el deportista aprenda por sí mismo a sacar partido a su máximo potencial y rendir de acuerdo con las exigencias de la competición. Sin embargo, nosotros pensamos que el coaching puede ir más allá del logro del máximo rendimiento, y que podría llevarse a otros ámbitos dentro de la actividad físicodeportiva.

En primer lugar, pensamos que tan apto es el uso del coaching en el deporte de élite como en cualquier otra práctica deportiva de nivel inferior, ya que el objetivo no es otro que el de hacer ver a la persona lo que realmente lleva dentro y puede explotar. Además, desde nuestro punto de vista, el coaching es un método perfectamente aplicable en la escuela, tanto en Educación Física como en el resto de materias. La idea de su aplicación en Educación Física va en sintonía con el autoconocimiento y la autoaceptación del alumnado, base sobre la cual intentaríamos construir una persona capaz de dejar a un lado los miedos y complejos y tener una actitud abierta hacia el aprendizaje, con voluntad de cambio, a la que no importe que las cosas al principio no salgan como a ella le gustaría y pudieran hacerle pasar por situaciones algo ridículas a ojos de los demás. Lo importante debería ser aprender de estos errores para poder enmendarlos en próximas ocasiones y aprender a divertirse realizando actividad física.

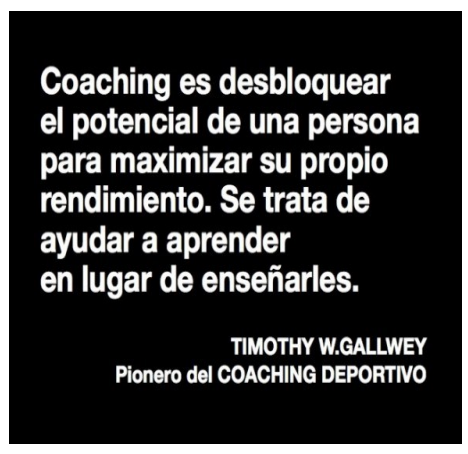




\section{In pionero en el coaching deportivo: W. Timothy Gallwey}

Gallwey es un autor que podríamos encuadrar dentro de los pioneros en el estudio y la publicación acerca del coaching deportivo. Uno de sus libros más conocidos es "The inner game of tennis", del cual hablaremos a continuación.

En el libro, este autor viene a decirnos que muchas veces somos nuestro peor enemigo. No es el adversario externo el que nos derrota sino nuestras propias dudas, nuestro propio miedo y nuestra falta de concentración. El Juego Interior del Tenis nos muestra la forma de superar estos obstáculos y de lograr un óptimo desempeño. Este libro está basado en el tenis, pero sus principios pueden aplicarse a cualquier tipo de actividad. Si practicas el tenis te enseñará a mejorar tu juego, a aprender con más rapidez y eficacia y a confiar más en la sabiduría natural de tu cuerpo. Si no lo practicas, te ayudará a desarrollar ciertas habilidades que te servirán para tener éxito en cualquier tipo de juego o en cualquier campo de actividad.

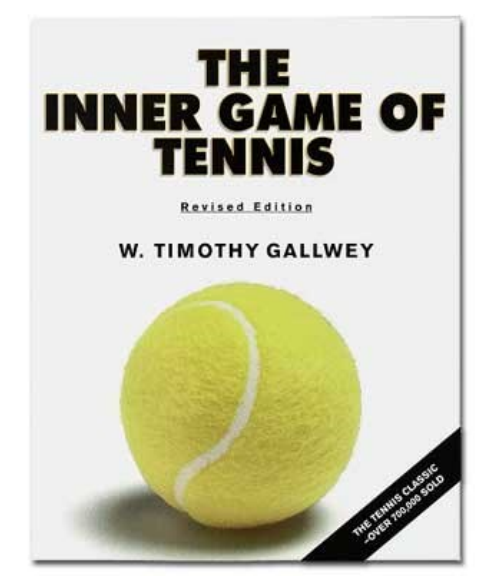

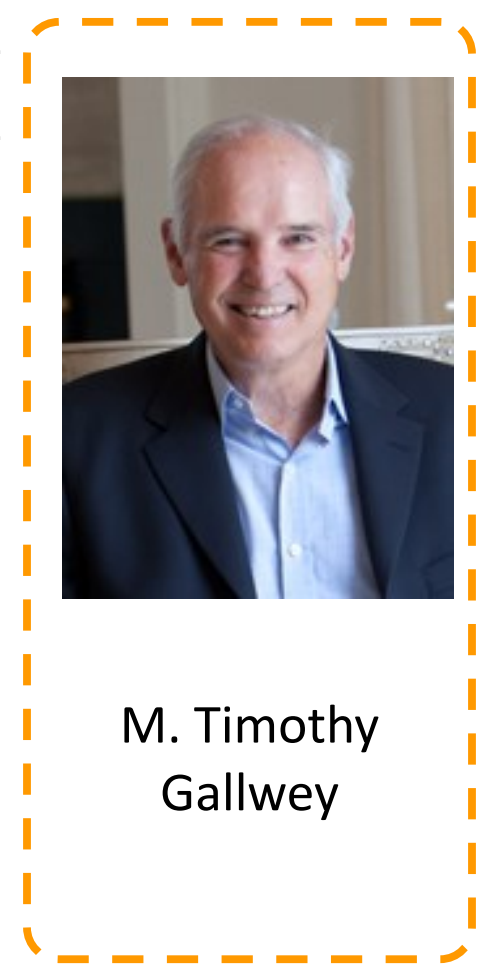

\section{Conclusiones}

El coaching es un método que tiene como objetivo llegar a ser mejores y más eficaces en los diferentes campos de nuestra vida, entre ellos en el deportivo. Para ello el coaching se centra en el autoconocimiento, ya que defiende que si nos conocemos mejor y nos relacionamos mejor con los demás, podremos actuar de forma más eficiente.

La relación que se debe establecer entre el coach y los deportistas debe ser la de facilitador del aprendizaje, de manera que a que a partir de su intervención, basada en preguntas, el deportista pueda obtener un mejor autoconocimiento, así como redescubrir su motivación; siendo necesario comenzar conociendo la visión que esa persona tiene de sí mismo , para a partir de ahí, establecer las estrategias a seguir. Esto será aplicable tanto en el ámbito deportivo, como en el profesional y sobre todo en el educativo, ya que un buen desarrollo de inteligencia emocional y autodescubrimiento durante la infancia será clave para el posterior éxito de nuestras vidas.

"Todo es posible en la medida que tú creas que es posible". Arturo Orantes 


\section{Para saber más...}

García-Naveira, A. "Aplicación profesional del coaching en el deporte: un estudio de caso único", en Cuadernos de Psicología del Deporte, vol. 13, 2, 101112:

http://8085-bddoc.csic.es.columbus.uhu.es/

detalles. $h$ tml; jsessionid $=2$ C6FC92B40A9B2D09440EDF6429F0A4A? $i d=740316 \& b d=I S O C \&$ tabla $=$ docu

Las Preguntas Mágicas del Coaching con PNL que logran cambios duraderos: https://www.youtube.com/watch?v=AgryNe36OLE

¿Qué es coaching?:

https://www.youtube.com/watch?v=UzdNDdOz-Ck

"Daría todo lo que sé, por la mitad de lo que ignoro". René Descartes.
Coaching - Juan Fernando Bou - Conferencia UVG Guatemala: https://www.youtube.com/watch?v=ygSEh5Ak-ic

An Association for Coaching Interview - Tim Gallwey: https://www.youtube.com/watch? $\mathrm{v}=\mathrm{q} 8 \mathrm{X} 0 \mathrm{v} 1 \mathrm{NgXgQ} \# \mathrm{t}=19$

Meya-Molina, A. Giménez-Sánchez, J (2014) "Coaching nutricional como herramienta para la adquisición de hábito alimentarios preventivos del cáncer", en Revista Española de Nutrición Humana y Dietética, 18(1): 35 - 44: dialnet.unirioja.es/descarga/articulo/4658797.pdf

Cantón, E. (2014) “¿El coaching es psicología? ¿Quién puede aplicar el Coaching y en qué entornos?”, en Información Psicológica, $n^{\circ} 107$, pp. 2-10. 


\section{Bibliogralía}

Blog David Priego "La Rueda de la Vida”:

http://davidprietov.blogspot.com.es/2013/03/la-rueda-de-la-vida.html

Blog Sergio Carrasco "Vivir mejor":

http://www.sergiocarrascomendez.com/

Fraile, A. (2013) "El coaching como estrategia pedagógica para la mejora del autoconocimiento de los educadores deportivos", en E-motion. Revista de Educación, Motricidad e Investigación , $n^{o} 1, p p .1-13$.

http://rabida.uhu.es/dspace/bitstream/handle/10272/8017/

El_coaching_como_estrategia.pdf?sequence $=2$

Galán, F.J. (2014) “¿Cómo definir objetivos con el método G.R.O.W.?”. Sitio web oficial de INTRAS:

http://www.intras.com.do/index.php?option $=$ com content\&view $=$ article $\&$ id $=243:$ icomodefinir-objetivos

García-Naveira, A. (2013) "Aplicación profesional del coaching en el deporte: un estudio de caso único" en Cuaderno de Psicología del Deporte, vol. 13, 2, 101-112:

http://scielo.isciii.es/pdf/cpd/v13n2/art10.pdf

Gutiérrez, D. (2011) “Coaching deportivo para entrenadores de fútbol ( $1^{\mathrm{a}}$ parte)” en FútbolTáctico, $n^{\circ}$ 47, pp. 55-63.

Gutiérrez, D. (2011) "Coaching deportivo para entrenadores de fútbol (2a parte)" en FútbolTáctico, $n^{\circ}$ 48, pp. 143-149.

Gutiérrez, D. (2011) "Coaching deportivo para entrenadores de fútbol ( $3^{\mathrm{a}}$ parte)" en FútbolTáctico, $n^{\circ}$ 49, pp. 113-117.

López, E. "La Escucha Activa. Diferencia entre oír y escuchar” http://www.coachingexitopersonal.com/coaching-exito-personal-de-vida/la-escuchaactiva-diferencia-entre-oir-y-escuchar/

Sans, M. (2012) “¿Qué es el coaching? Sus orígenes, definición, distintas metodologías y principios básicos de actuación de un coach " en 3c Empresa: investigación y pensamiento crítico, Vol. 1, N $\mathrm{N}^{\mathrm{O}}$ : http://dialnet.unirioja.es/servlet/articulo? codigo $=4817930$

Sitio web oficial de la Escuela de Formación Integral en Coaching: http:/lefic.es/conoce-efic/colaboradores/sergio-carrasco/

Sitio web oficial de la International Coach Federation (ICF):

http://www.icf-es.com/mwsicf/sobreicf/definicion-coaching-icf-espana

Youtube.com:

https://www.youtube.com/watch? $v=$ Kidd 1 KpyHYY 